\title{
Short wave instability of co-extruded elastic liquids with matched viscosities
}

\author{
H.J. Wilson ${ }^{1}$, J.M. Rallison \\ DAMTP, University of Cambridge, Silver Street, Cambridge, CB3 9EW, UK
}

\begin{abstract}
The stability of channel flow of coextruded elastic liquids having matched viscosities but a jump in elastic properties is studied. Inertia and surface tension are neglected. A short wave disturbance is found, confined near the interface, whose growth rate is independent of wavelength. For dilute Oldroyd-B fluids this disturbance is unstable for any non-zero jump in normal stresses, and has a maximum growth rate for intermediate levels of elasticity in the two fluids. When one or other fluid is highly elastic the growth rate falls. In the concentrated limit (a UCM fluid), the disturbance is unstable only for a finite range of normal stress jumps, and is restabilised if one fluid is much more elastic than the other. Because the short wave disturbance is localised, the results apply for any steadily sheared interface across which the normal stress jumps. The results are confirmed for moderate parameter values by means of a full numerical solution for a three-layer planar flow.
\end{abstract}

Keywords: Coextrusion; Core-annular flow; Short wave; Instability; Oldroyd-B; UCM; Normal stresses

\section{Introduction}

We consider in this paper the coextrusion of two elastic liquids. Our principal aim is to identify the physical processes which generate and inhibit instability of the interface between the fluids.

When there is a jump in viscosity, an instability is present even for Newtonian liquids [10] and we isolate the central non-Newtonian aspect by considering a case where viscosity varies continuously across the interface, and only normal stresses jump. Additionally inertial effects can destabilise Newtonian pipe and channel flows, and thus we restrict attention here to the simplest case where inertia is negligible.

For long wavelength disturbances Hinch et al. [3] provide a mechanism for, and calculation of, the growth of disturbances resulting from normal stresses. This operates only when the annulus is more elastic than the core, and has zero growth rate as the wavelength tends to infinity. Our principal focus here then is to understand the opposite limit of short waves.

We find that in the absence of surface tension, interfaces between different Oldroyd-B fluids are always unstable to short-wave disturbances except at high concentration and high elasticity, that the growth rate becomes independent of wavelength in this limit, and that the symmetry of

\footnotetext{
${ }^{1}$ Corresponding author. Fax: + 441223337918.
} 
the disturbance (whether varicose or sinuous, whether planar or axisymmetric) is irrelevant to the mechanism at work. The central conclusion from our analysis is therefore that surface tension needs to be sufficiently large if elastic interface disturbances are to be damped. We examine the importance of surface tension effects in Section 6.

Related work on this phenomenon has been performed by Su and Khomami, who have studied analytically and numerically the stability of two-layer die flow [8, 9], and Renardy, who tackled the short-wave behaviour of two-layer Couette flow [7]. More recently, Laure et al. [5] have studied two- and three-layer Poiseuille flow of Oldroyd-B fluids, with long to moderate wavelength perturbations, incorporating the effects of surface tension, viscosity stratification, density stratification and inertia.

We formulate the problem for arbitrary interface disturbances in Section 2 which provides a numerical problem against which our short-wave asymptotics in Section 3 can later be tested. Limits of high and low relative elasticity are identified in Section 4 and conclusions are given in Section 6.

\section{Statement of the problem}

The basic geometry is a planar channel flow, sketched in Fig. 1, with different Oldroyd-B fluids occupying the regions $\kappa<|y|<1,|y|<\kappa$ respectively.

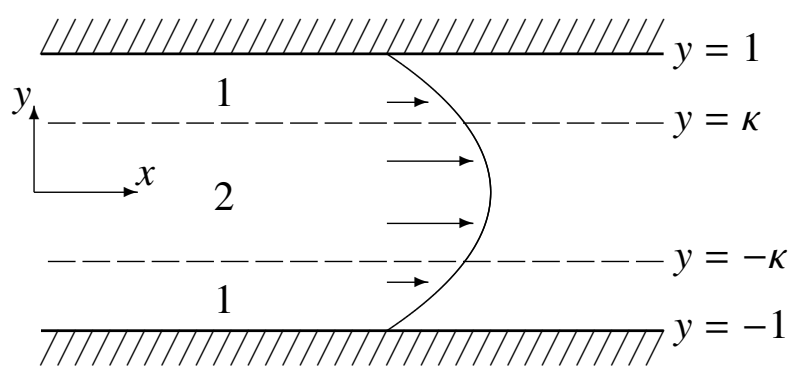

Figure 1: Flow geometry.

We suppose that both fluids have the same (constant) shear viscosity, but different normal stress coefficients. A base Poiseuille flow is then possible throughout. It is convenient to scale lengths by the half-width of the channel, $d$, velocities by that on the centreline of the channel, $U$, and stresses by $\mu U / d$ where $\mu$ is the solvent viscosity.

Inertia is neglected, as is surface tension at the interface between the two fluids. The nondimensional groups that remain are then:

$\kappa \quad$ the relative width of the inner fluid;

$c$ the ratio of the elastic zero-shear viscosity contribution to the solvent contribution: the concentration of polymer;

$W_{i}$ the Weissenberg number in fluid $i$ : the ratio of fluid relaxation time to a typical flow time.

Conservation of mass and momentum then give

$$
\begin{aligned}
\nabla \cdot \boldsymbol{u} & =0 \\
\nabla \cdot \boldsymbol{\sigma} & =0
\end{aligned}
$$


where $\boldsymbol{u}$ is the velocity field and $\boldsymbol{\sigma}$ the stress tensor. The constitutive equation for each Oldroyd-B fluid may be written in the form

$$
\begin{gathered}
\boldsymbol{\sigma}=-p \boldsymbol{I}+\left(\nabla \boldsymbol{u}+\nabla \boldsymbol{u}^{\top}\right)+\frac{c}{W} \boldsymbol{A}, \\
\frac{D \boldsymbol{A}}{D t}=\nabla \boldsymbol{u}^{\top} \cdot \boldsymbol{A}+\boldsymbol{A} \cdot \nabla \boldsymbol{u}-\frac{1}{W}(\boldsymbol{A}-\boldsymbol{I}) .
\end{gathered}
$$

The concentration parameter, $c$, is matched in the two fluids, so each has the same shear viscosity, $1+c$. The two fluids have different Weissenberg numbers in order for the interface to have any effect, otherwise there is effectively only one fluid present. We call these $W_{1}$ and $W_{2}$.

The equations of motion are obeyed within each fluid, and at the walls of the channel there is a no-slip, no-penetration condition. At the interface between the two fluids, velocity is continuous and so is the traction $\boldsymbol{\sigma} \cdot \boldsymbol{n}$, where $\boldsymbol{n}$ is the normal to the interface.

\subsection{Base state}

A solution of the equations which satisfies the boundary conditions is Poiseuille flow,

$$
\boldsymbol{u}=\left(\begin{array}{cc}
1-y^{2}, & 0
\end{array}\right) \text {. }
$$

The elastic stresses in the base state are given for $i=1,2$ as

$$
\begin{gathered}
\boldsymbol{A}_{i}=\left(\begin{array}{cc}
1+8 W_{i}^{2} y^{2} & -2 W_{i} y \\
-2 W_{i} y & 1
\end{array}\right), \\
\boldsymbol{\Sigma}_{i}=\left(\begin{array}{cc}
-P_{i}+c / W_{i}+8 c W_{i} y^{2} & -2 y(1+c) \\
-2 y(1+c) & -P_{i}+c / W_{i}
\end{array}\right), \\
P_{i}=P_{\infty}+c / W_{i}-2(1+c) x
\end{gathered}
$$

It is clear that $\Sigma_{11}$ has a discontinuity of magnitude $8 c \kappa^{2}\left|W_{1}-W_{2}\right|$ at each interface, resulting in this nondimensional form from the quadratic growth in normal stress for Oldroyd-B fluids. This jump is permissible only because the interfaces are parallel to the $x$-axis.

The fundamental mechanism of the elastic instability (as shown in [3]) is that the tilting of this interface exposes the jump in $\Sigma_{11}$ and this drives a perturbation flow which may in its turn cause the perturbation to grow.

\subsection{Perturbation flow}

We impose a perturbation on the base state by moving the interfaces to $y= \pm \kappa \pm \delta e^{i k x-i \omega t}$, where $\delta$ is an infinitesimally small amplitude, and linearise the governing equations about the base state. A sinuous perturbation is given by $y= \pm \kappa+\delta e^{i k x-i \omega t}$, and a varicose perturbation is $y= \pm\left(\kappa+\delta e^{i k x-i \omega t}\right)$. Factoring out the $x$ and $t$ dependence of the perturbation quantities, and denoting $\mathrm{d} / \mathrm{d} y$ by $D$, we obtain

$$
\left(D^{2}-k^{2}\right)^{2} \psi+\frac{c}{W}\left(D^{2}+k^{2}\right) a_{12}+i k \frac{c}{W} D\left(a_{11}-a_{22}\right)=0
$$




$$
\begin{gathered}
\left(-i \omega+i k\left(1-y^{2}\right)+\frac{1}{W}\right) a_{11}=16 i k W^{2} y \psi+2 i k\left(1+8 W^{2} y^{2}\right) D \psi-4 W y D^{2} \psi-4 y a_{12} \\
\left(-i \omega+i k\left(1-y^{2}\right)+\frac{1}{W}\right) a_{12}=-2 i k W \psi+k^{2}\left(1+8 W^{2} y^{2}\right) \psi+D^{2} \psi-2 y a_{22} \\
\left(-i \omega+i k\left(1-y^{2}\right)+\frac{1}{W}\right) a_{22}=-4 k^{2} W y \psi-2 i k D \psi
\end{gathered}
$$

where $\psi$ is the two-dimensional stream function and $a$ the perturbation to $A$.

Eq. (9) here is the vorticity equation, and Eqs. (10)-(12) arise from the evolution of $\boldsymbol{A}$.

If the perturbation is sinuous, the $x$-velocity at the centre is unchanged under the transformation $y \rightarrow-y$, so $\psi$ must be an odd function of $y$. Alternatively, for a varicose perturbation, there must be no $y$-velocity on the centreline of the channel, so $\psi$ is even. Either of these provides two boundary conditions at $y=0$ and allows us to consider only the upper half channel. We have therefore:

$$
\begin{aligned}
& \text { Sinuous } D \psi(0)=D^{3} \psi(0)=0 \\
& \text { Varicose } \psi(0)=D^{2} \psi(0)=0
\end{aligned}
$$

The no-slip, no-penetration boundary condition still holds for the perturbation flow, which gives two more boundary conditions at $y=1$.

$$
\psi(1)=D \psi(1)=0
$$

At the interface $y=\kappa$, velocity $(\psi$, and $D \psi)$ is continuous and so is the traction, so that

$$
\begin{gathered}
{\left[\left(D^{2}+k^{2}\right) \psi+\frac{c}{W_{i}} a_{12}-8 i k c \kappa^{2} W_{i} \delta\right]=0,} \\
{\left[D\left(D^{2}-3 k^{2}\right) \psi+\frac{c}{W_{i}} D a_{12}+i k \frac{c}{W_{i}}\left(a_{11}-a_{22}\right)\right]=0}
\end{gathered}
$$

where the square brackets denote a jump across $y=\kappa$.

We have finally a kinematic boundary condition stating that the interface is a material surface:

$$
\left(-i \omega+i k\left(1-\kappa^{2}\right) \delta=-i k \psi(\kappa)\right.
$$

These equations and boundary conditions provide a well-posed problem in which we can impose the wavenumber $k$ and seek $\omega$ : in other words Eqs. (9)-(13) and (14)-(17) give the dispersion relation $\omega(k)$. The imaginary part of $\omega$ is the growth rate. We show numerical results in Section 5 but first consider the $k \rightarrow \infty$, asymptotics. 


\section{Short wave limit}

The long-wave limit is considered in [3]. Here we concentrate on short waves.

The Oldroyd-B fluid has no intrinsic lengthscale. Thus in the short-wave limit the wavelength $k^{-1}$ becomes the only lengthscale defining the flow. At leading order as $k \rightarrow \infty$ the perturbation is confined within $O\left(k^{-1}\right)$ of the interface. So the walls of the channel, the perturbation of the other interface and the variation in base shear-rate with $y$ have no effect. The results therefore apply to any interface (without surface tension): there is no distinction here between sinuous or varicose, planar or axisymmetric disturbances, all of which have the same growth rate.

If we move to a frame of reference travelling with the interface, i.e. at speed $1-\kappa^{2}$, move the level $y=0$ to the interface, and rescale all lengths with $k$, we obtain at leading order the equations for a perturbation to unbounded simple shear flow of two fluids having a constant shear rate $-2 \kappa$ :

$$
\begin{gathered}
\left(D^{2}-1\right)^{2} \psi+\frac{c}{W}\left(D^{2}+1\right) a_{12}+i \frac{c}{W} D\left(a_{11}-a_{22}\right)=0 \\
\left(-i \omega-2 i \kappa y+\frac{1}{W}\right) a_{11}=2 i\left(1+8 W^{2} \kappa^{2}\right) D \psi-4 W \kappa D^{2} \psi-4 \kappa a_{12} \\
\left(-i \omega-2 i \kappa y+\frac{1}{W}\right) a_{12}=\left(1+8 W^{2} \kappa^{2}\right) \psi+D^{2} \psi-2 \kappa a_{22} \\
\left(-i \omega-2 i \kappa y+\frac{1}{W}\right) a_{22}=-4 W \kappa \psi-2 i D \psi
\end{gathered}
$$

The following functions must be continuous at the interface $y=0$ :

$$
\begin{gathered}
\psi, D \psi, \\
\left(D^{2}+1\right) \psi+\frac{c}{W} a_{12}-8 i c \kappa^{2} \delta W,
\end{gathered}
$$

and

$$
D\left(D^{2}-3\right) \psi+\frac{c}{W} D a_{12}+i \frac{c}{W}\left(a_{11}-a_{22}\right)
$$

and the kinematic condition reduces to

$$
\omega \delta=\psi(0),(25)
$$

while the perturbation decays away from the interface:

$$
\psi(y) \rightarrow O \text { as } y \rightarrow \pm \infty .
$$

We note that $k$ has disappeared from the problem and thus this set of equations gives instability at a growth rate that is constant as $k \rightarrow \infty$. The symmetry of the unbounded system means that exchanging $W_{1}$ and $W_{2}$ is equivalent to reversing the direction of shear, which reverses the convective part of $\omega$ but does not change the growth rate. Note that neither $\omega$ nor any of the dimensionless fluid parameters $c, W_{1}, W_{2}$ has been rescaled in this reformulation; $\omega$ here is the same as in Section 2.2 except for the subtraction of a term $\left(1-\kappa^{2}\right) k$ inherent in the change of frame of reference. 
For the rest of the study of short waves, since we are interested in unbounded Couette flow, for convenience we have put the interface at the representative point $\kappa=1 / 2$, i.e. a shear rate of -1 at the upper interface. The geometry is simple two-dimensional unbounded shear flow of superposed fluids, with the interface parallel to the direction of flow.

Even this reduced problem requires numerical solution (because of the advective terms proportional to $y$ in Eqs. (19)-(21)), but $\omega$ is a function only of $c, W_{1}$ and $W_{2}$ and the central features may be identified by examining extreme cases.

\section{Asymptotic limits}

\subsection{Concentrated solutions, $c \rightarrow \infty$}

As the concentration parameter $\mathrm{c}$ tends to $\infty$ the Oldroyd-B fluid effectively loses its solvent contribution giving the Upper-Convected Maxwell fluid (UCM). This fluid was studied in Couette flow by Renardy [7] and the stability problem solved analytically. The neutral stability curves in the $W_{1}-W_{2}$ plane are shown in Fig. 2 . We note that the interface is stable if either $W_{1}=W_{2}$ or $W_{1}$ and $W_{2}$ are widely different.

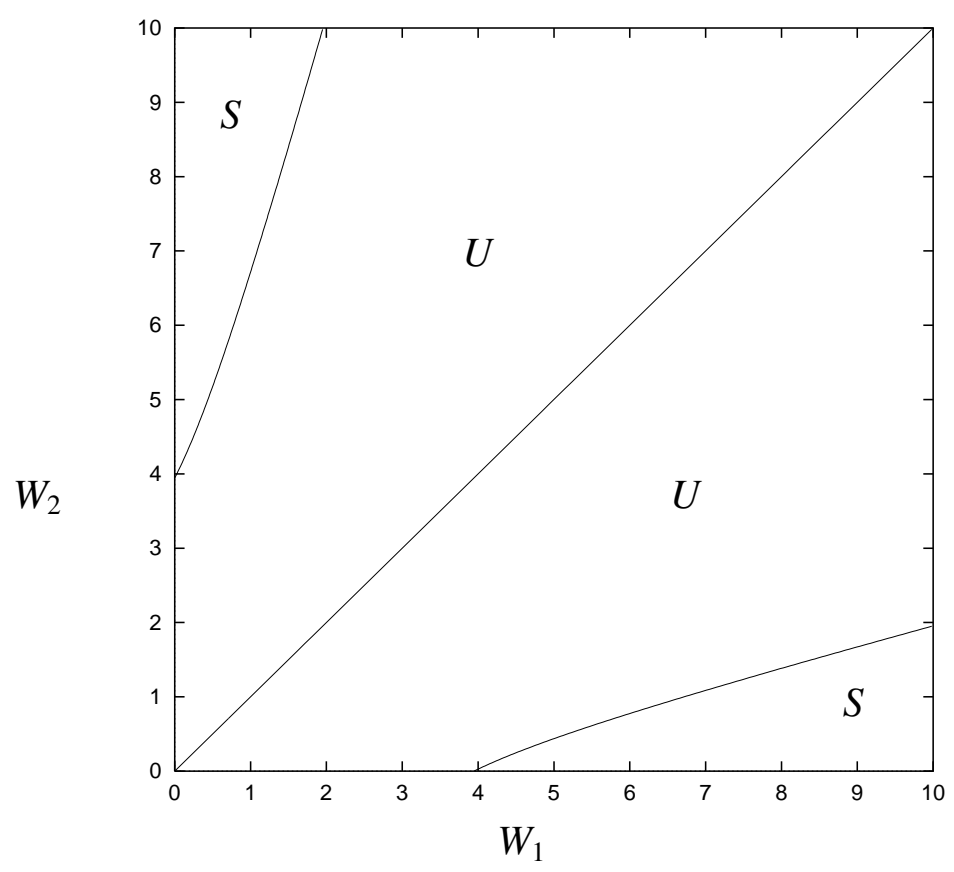

Figure 2: Neutral stability curves for $c=\infty, k=\infty, \kappa=1 / 2$. $U$, unstable; $S$, stable [7]. There is stability if $W_{1}=W_{2}$ or if $W_{1}$ and $W_{2}$ are very different from each other. Elsewhere the interface is unstable.

\subsection{Dilute solution, $c \rightarrow 0$}

In the dilute limit, both fluids are nearly Newtonian in character, and the effect of elasticity appears only at order $c^{3}$. All the perturbation quantities may be expanded as powers of $c$ and the 
eigenvalue problem solved iteratively using 'Maple' [1]. The eigenvalue is:

$$
\begin{aligned}
& \omega=-\frac{1}{4} c^{2}\left(W_{1}-W_{2}\right)\left(W_{1}+W_{2}\right) \\
& +\frac{1}{8} i c^{3}\left(W_{1}-W_{2}\right)\left(W_{1}+W_{2}\right)\left(W_{1}-W_{2}-2 i+2\left(W_{1}-i\right) \varepsilon\left(2 i / W_{1}\right)-2\left(W_{2}+i\right) \varepsilon\left(-2 i / W_{2}\right)\right)+O\left(c^{4}\right),
\end{aligned}
$$

where

$$
\varepsilon(x)=\int_{0}^{\infty} \frac{e^{-x t}}{1+t} \mathrm{~d} t
$$

is the exponential integral function.

At leading order in $c$, this shows neutral stability (as expected) when $W_{1}=W_{2}$, but instability for all other $W_{1}, W_{2}$. This is qualitatively different from Renardy's result for UCM above.

Because the concentrated limit is stable for widely separated values of $W_{1}$ and $W_{2}$, there must exist some critical concentration $c_{\text {crit }}$ above which restabilisation occurs. We define this concentration by looking at the behaviour of $\omega$ as $W_{2}$ increases with $W_{1}=0$; for $c<c_{\text {crit }}$ the imaginary part of $\omega$ is always positive, whereas for $c>c_{\text {crit }}$ the growth rate is negative as $W_{2} \rightarrow \infty$. Numerical results show $c_{\text {crit }} \approx 8$ : above this value we can find pairs of Weissenberg numbers for which the flow is stable to perturbations of short wavelength, and indeed for these values the flow is found (using the methods of Section 5) to be stable to all wavelengths provided the inner fluid is more elastic: for example, at $c=100, \kappa=0.5, W_{1}=0$, the flow is stable for all $W_{2}>4.25$.

\subsection{The retarded motion limit, $W_{1}, W_{2} \rightarrow 0$}

If both Weissenberg numbers tend to zero keeping their ratio constant, we can expand the streamfunction, stretches and eigenvalue as Poincaré series for arbitrary $c$, and obtain

$$
\begin{aligned}
\omega= & -\frac{1}{4} \frac{c^{2}}{(1+c)^{2}}\left(W_{1}-W_{2}\right)\left(W_{1}+W_{2}\right) \\
& +\frac{1}{16} \frac{c^{3}}{(1+c)^{4}}\left(W_{1}-W_{2}\right)\left(W_{1}+W_{2}\right)\left(3\left(W_{1}^{2}+W_{2}^{2}\right)+2 c\left(2\left(W_{1}^{2}+W_{2}^{2}\right)-W_{1} W_{2}\right)\right) \\
& +\frac{i}{32} \frac{c^{3}}{(1+c)^{5}}\left(W_{1}-W_{2}\right)^{2}\left(W_{1}+W_{2}\right)\left(\left(c^{2}+3 c+1\right)\left(3\left(W_{1}^{2}+W_{2}^{2}\right)+4 W_{1} W_{2}\right)+W_{1}^{2}+W_{2}^{2}\right) \\
& +O\left(W_{i}^{6}\right) .
\end{aligned}
$$

We note for these slow weak flows that the growth of the disturbance is inhibited by the total zeroshear viscosity $1+c$ (not just the solvent viscosity). Furthermore, because in the limit $W_{i} \rightarrow 0$ there is no elasticity, the growth rate must vanish as $W_{i} \rightarrow 0$. Our result for $\omega$ is consistent with this requirement, i.e. this is not a spurious instability violating the slow flow expansion.

In a slow flow expansion, any simple fluid may be regarded as an $n$ th-order fluid with an error of $O\left(W^{n}\right)$. Surprisingly, perhaps, the first term giving a non-zero growth rate is $O\left(W_{i}^{5}\right)$, thus we anticipate that a fourth-order fluid would not be able to capture this behaviour. We expect that a sixth-order fluid would be needed and although such a result would be universal, the result is unlikely to be useful in view of the large number of undetermined constants. 


\subsection{Slow relaxation, high elasticity, $W_{2} \rightarrow \infty$}

In the limit of large Weissenberg number, at leading order the stretches $a_{11}$ and $a_{12}$ in Eqs. (19)(21) scale as $W^{2}$ (for $y=O(1)$ ) so that the vorticity equation (18) loses its highest derivative. Thus the limit is singular, suggesting the appearance of boundary layers. In order to simplify the problem, we consider the case where a Newtonian fluid occupies one half-plane $y>0$ (which is equivalent to setting $W_{1}=0$ ) with elastic forces only in $y<0$.

Detailed analysis reveals that two lengthscales arise in the elastic fluid near the interface. First there is a boundary layer of width $1 / W_{2}$, across which the pressure is approximately constant but the velocities $\psi, D \psi$ and the shear stress $\sigma_{12}$ change by a factor of order $W_{2}$. Within this layer there is a balance between the growth (or decay) of the perturbation and the elastic relaxation terms in the evolution of the stretches. Second, on a lengthscale of order unity (remembering that all lengthscales have been rescaled by $k^{-1}$ here) there arises an incompressible elastic deformation in which elastic forces dominate viscous forces so that $\psi \sim y e^{-y}$ and $\nabla^{2} p=0$. The advection due to shear remains important.

Typical streamlines of the perturbation flow (calculated numerically) are shown in Figs. 3 and 4 , showing the elastic boundary layer.

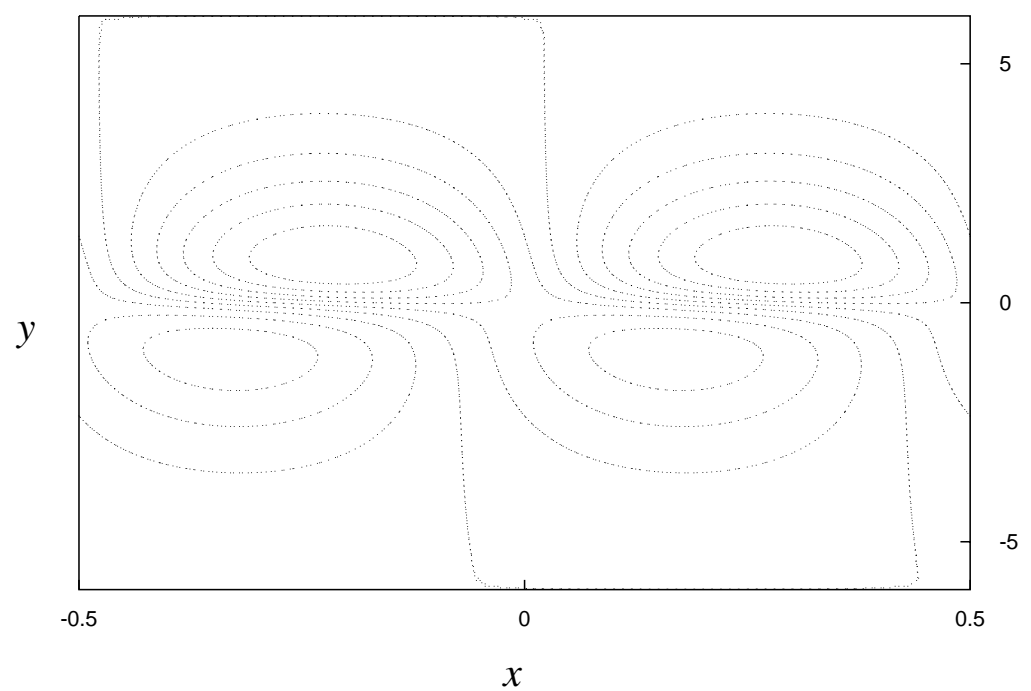

Figure 3: Streamlines of perturbation to Couette flow; unstable case. $c=0.2, W_{2}=5$.

The eigenvalue $\omega$ is of order $1 / W_{2}$ but its sign is not obvious, and needs to be determined numerically as a function of $c$. As noted above we find that the growth rate changes sign at $c \approx 8$.

This limit is difficult to calculate and the result may be surprising because although the jump in $\Sigma_{11}$ is large $\left(W_{1}-W_{2}\right.$ is large) the growth rate $\omega$ is small $\left(O\left(1 / W_{2}\right)\right)$. The reason is that the highly elastic fluid strongly resists the perturbation (the boundary layer acting rather like an extra surface tension). It greatly reduces the growth rate and can (for sufficiently high concentrations $c>c_{\text {crit }}$ ) prevent the instability. 


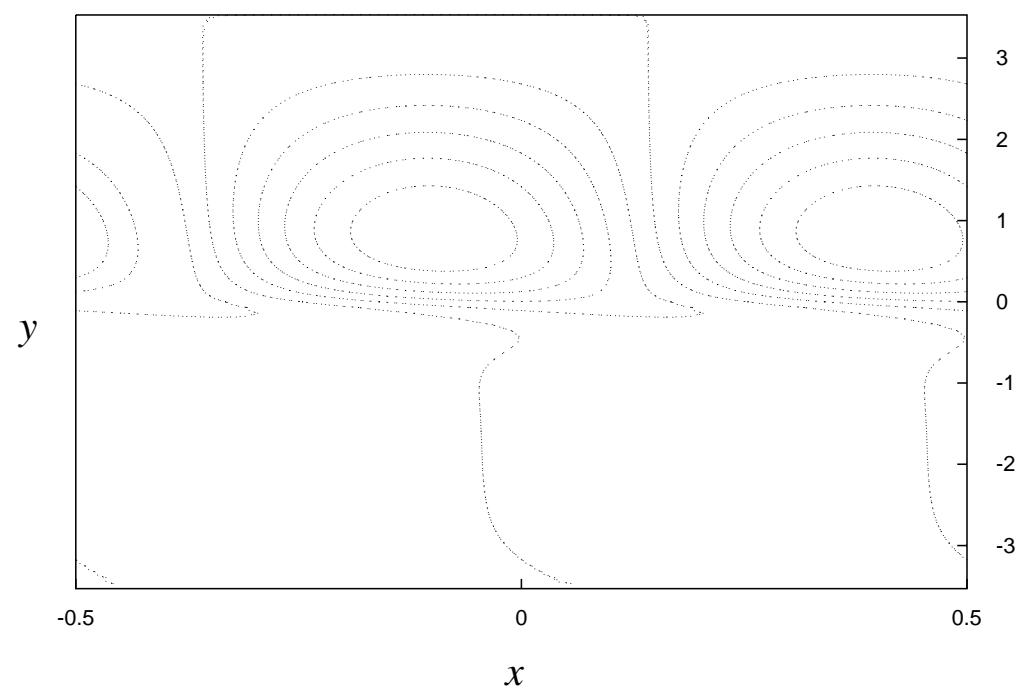

Figure 4: Streamlines of perturbation to Couette flow; stable case. $c=\infty(\mathrm{UCM}), W_{2}=5$.

\subsection{Arbitrary $W$}

We conclude from the asymptotic cases examined above that for fixed $c<c_{\text {crit }}$, the growth rate of disturbances will be small (and positive) if $W_{1}-W_{2}$ is small (since this reduces the driving force) and small in magnitude if $W_{1}-W_{2}$ is large (since the bulk elastic resistance then rises). Thus there is an intermediate value of $W_{1}-W_{2}$ when growth is largest, as shown in Fig. 5.

\section{Numerical results}

Away from the limits $k \rightarrow 0$ [3] and $k \rightarrow \infty$ (Section 3), a numerical study was used.

The system Eqs. (9)-(13) and (14)-(17) is a fourth-order differential equation for $\psi(y)$ with three point boundary conditions. Given a fixed $\omega$ and values of $\psi$ and its first three derivatives at either the channel wall $(y=1)$ or the centreline $(y=0)$, the vorticity equation (9) may be integrated numerically by fourth-order Runge-Kutta to give $\psi$ and its derivatives at the interface.

There are four potentially nonzero forcings arising from the wall and centreline boundary conditions (viz $D^{2} \psi(1), D^{3} \psi(1)$, and either $\psi(0)$ and $D^{2} \psi(0)$ or $D \psi(0)$ and $D^{3} \psi(0)$ ). Following Ho and Denn [4], we impose an estimated $\omega$ and form a basis by considering each boundary condition in turn. We set, for example, $D^{3} \psi(1)=0, D^{2} \psi(1)=1$, and integrate from the wall to the interface. We repeat the process for $D^{3} \psi(1)=1, D^{2} \psi(1)=0$; any streamfunction must in the outer fluid be a linear combination of these two. The inner fluid is treated similarly, and the four interfacial jump conditions (defining $\delta$ through the kinematic boundary condition Eq. (17)) become a matrix equation of the form

$$
M \boldsymbol{b}=0
$$




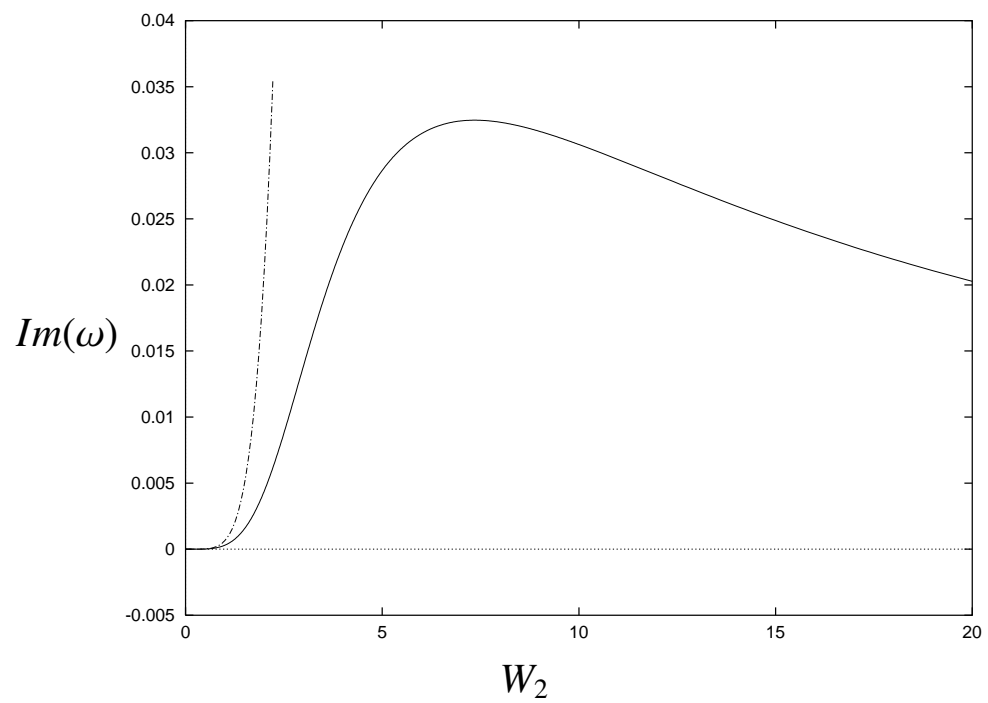

Figure 5: Growth rate against $W_{2}$ when $c=0.2, W_{1}=0$; the dotted curve is the asymptote found for the theory of 4.3 . As $W_{2} \rightarrow \infty$, the growth rate is proportional to $1 / W_{2}$ but the coefficient is not known analytically.

where $\boldsymbol{b}$ is the vector of coefficients of each of the four linearly independent functions obtained above. Thus the problem is reduced to finding an $\omega$ such that the determinant of $\boldsymbol{M}(\omega)$ is zero. A Newton-Raphson procedure is used.

Representative behaviours of the growth rate of the most unstable disturbances with varying wavenumber $k$ are shown in Figs. 6-9.

If the outer fluid is more elastic, then long waves are unstable, and the sinuous mode is the more unstable for long waves [3] but can be overtaken by the varicose mode at some wavenumber of order unity. The modes have the same behaviour for very short waves. Note that in some cases the formation of boundary layers near the interface leads to numerical difficulties at high wavenumbers: an asymptote to the known short-wave limit is shown.

If the outer fluid is less elastic, then long waves are stable, and the behaviour is more complicated. The least stable mode at long waves is again that identified by Chen [2], but continuation of this mode to high wavenumbers does not necessarily give the least stable short-wave mode. Similarly, continuation of sinuous and varicose modes may also lead to different roots of the short-wave problem. A selection of the typical growth rate curves is plotted in Figs. 8 and 9. Note that when one of the Weissenberg numbers is zero, there is a mode for which long waves decay at rate $1 / W$, i.e. the relaxation rate of the elastic fluid. This corresponds to an elastic wave confined in the elastic fluid close to the interface (analysis of Eqs. (9)-(13) and (14)-(17) reveals a boundary layer of width $k$ ) which relaxes at the molecular relaxation rate (we are grateful to one of the referees for pointing this out).

For $c$ small (Fig. 8) the most unstable disturbances are either short wave, or have $k$ of order unity, i.e. the disturbance wavelength is comparable with the channel width. Thus the long-wave analysis, though sufficient to demonstrate instability, may be a poor predictor of the type, wavelength and growth rate of instability seen experimentally [6].

For $c$ large (Fig. 9), beyond some critical value of $k$ our Newton-Raphson routine is able to find 


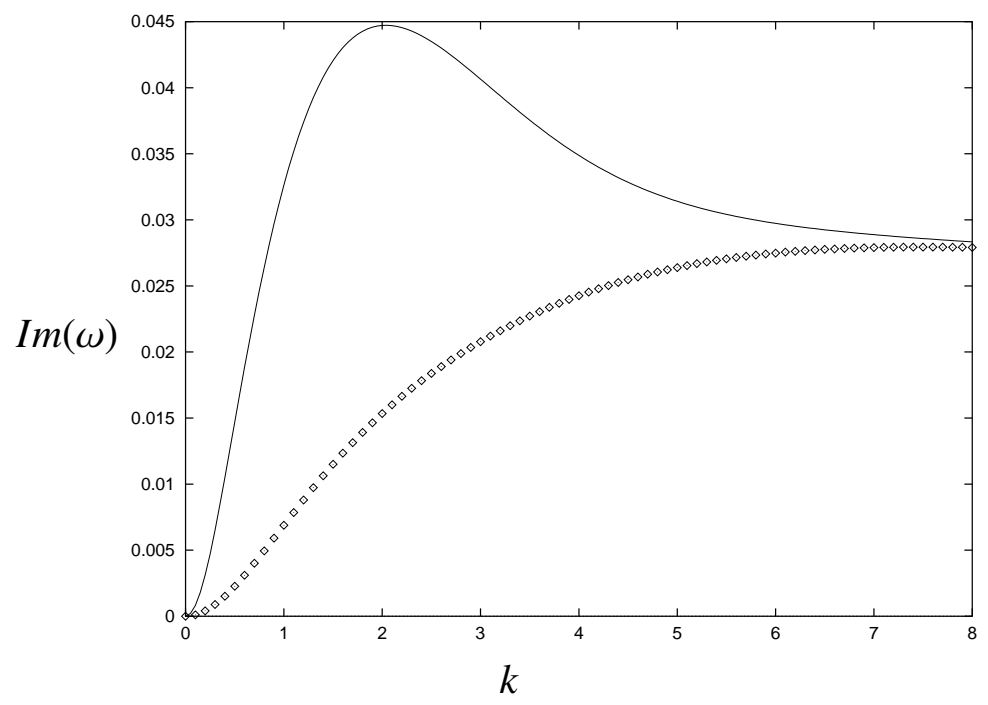

Figure 6: Growth rates plotted against $k$ for a case where both long and short waves are unstable, $c=0.2, \kappa=0.5$, $W_{1}=4, W_{2}=0$. Outer fluid more elastic, fastest growing mode is sinuous with $k \sim 2$. The solid curve is the sinuous mode, the points show the varicose mode.

only two roots of the dispersion relation for varicose modes. The Renardy analysis [7] for UCM as $k \rightarrow \infty$ gives a quintic polynomial for $\omega$ having five distinct roots.

We are able to find the least stable branch for sinuous modes, but not (for large finite $k$ ) in the varicose case. It is tempting to suppose that a new branch of varicose solutions appears beyond the critical value of $k$ corresponding to two of the Renardy modes, but at our level of numerical accuracy we are not able adequately to resolve the boundary layers that are present for high $k$ and high $W$ so as to decide this question.

\section{Conclusions}

We have investigated the stability to linear perturbations of a two-fluid planar Poiseuille flow, matching all the fluid properties except elasticity, and neglecting inertia and surface tension.

For dilute Oldroyd-B fluids, short waves are unstable over the full range of Weissenberg numbers $W_{1} \neq W_{2}$. Near the UCM limit however there is instability for only a finite range of $W_{1}-W_{2}$. The instability occurs for small $W$ at $O\left(W^{5}\right)$ so the phenomenon should not be limited to Oldroyd$B$ fluids. As the wavelength tends to zero, the growth rate remains finite.

The short-wave behaviour of the system is independent of the type of perturbation (sinuous or varicose) and of which fluid forms the core and which the annulus. Since the long-wave stability characteristics are reversed by interchanging the core and annulus $([3,5])$ short and long wave limits are fundamentally different from one another. In most cases, the short waves are unstable, and, having finite growth rates, are predicted to grow faster than the long waves, whose growth rate is small. In most cases the largest growth rate occurs for some moderate wavelength comparable with the channel width.

A vital ingredient neglected in our short wave analysis is surface tension. A dimensionless group that measures the relative importance of normal stress and surface tension effects is an 


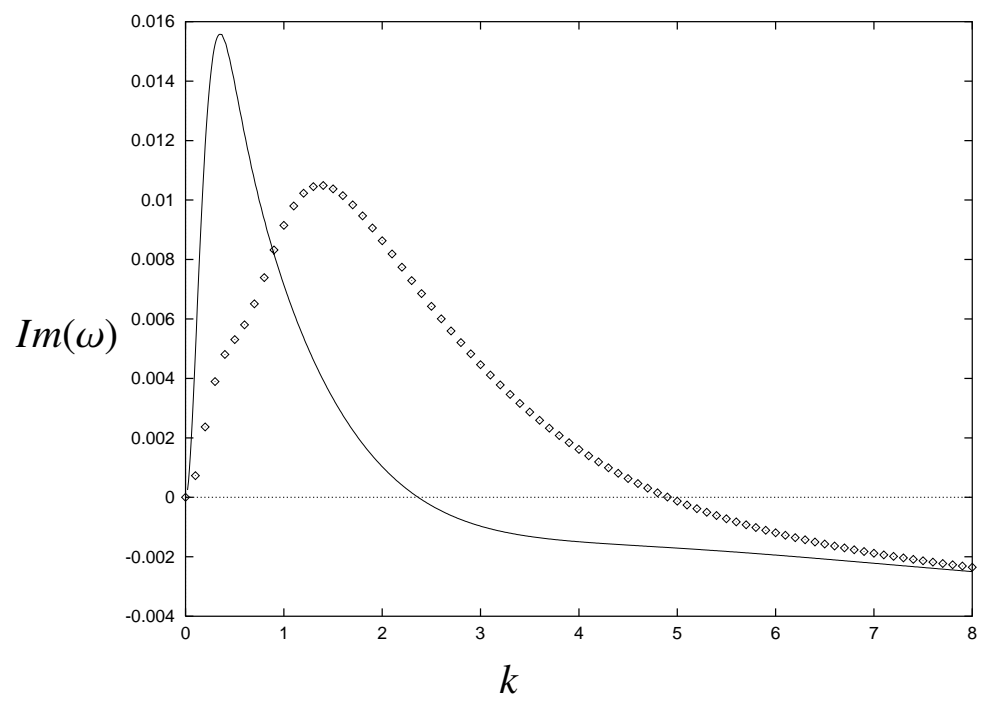

Figure 7: Growth rates plotted against $k$ for a case where long waves are unstable and short stable, $c=\infty$ (UCM), $\kappa=0.5, W_{1}=5, W_{2}=0$. Outer fluid more elastic, fastest growing mode is sinuous with $k \sim 0.5$. The first peak is the sinuous mode, and the points show the varicose mode.

elastic capillary number

$$
\frac{N_{1}}{T k}=W \frac{\mu \dot{\gamma}}{T k}
$$

where $k$ is the wavenumber of the perturbation at the interface, $T$ the surface tension coefficient, and $N_{1}$ the jump in the first normal stress difference. $W$ is the Weissenberg number, $\dot{\gamma}$ the shear rate at the interface and $\mu$ the shear viscosity.

For viscous polymeric liquids with $\mu \sim 1 \mathrm{P}$, surface tension coefficient $T \sim 10$ dyne $\mathrm{cm}^{-1}$ and shear rates $\dot{\gamma} \sim 1 \mathrm{~s}^{-1}$, then for Weissenberg numbers of order unity this imposes the strong requirement that the wavelengths considered should exceed $10 \mathrm{~cm}$. Since however the elastic capillary number scales with $\dot{\gamma}^{2}$, at shear rates of $10^{2} \mathrm{~s}^{-1}$, we need only the wavelength in excess of $10^{-3} \mathrm{~cm}$. In a channel of width $1 \mathrm{~cm}$ this value of $k$ lies on the short-wave plateau of Figs. 6-9.

The mechanism of all the instabilities here is the jump in $N_{1}$. This variation in $N_{1}$ is present also in elastic liquid flows where $N_{1}$ varies continuously across the channel (and for which surface tension vanishes). It may therefore be expected that continuous stratification may also generate instability. We intend to follow up this observation in a later paper.

\section{Acknowledgements}

This work was assisted by the SERC 'Computational Science Initiative' Grant GR/H57585 and HJW was supported by a Research Studentship from the EPSRC. JMR is also grateful for helpful discussions at Stanford supported by NATO Research Grant 941212.

\section{References}

[1] B.W. Char, K.O. Geddes, G.H. Gonnet, B.L. Leong, M.B. Monagan, S.M. Watt, Maple V Library/Language Reference Manual, Maple Publishing, Springer-Verlag and Waterloo, Maple Pub., 1991. 


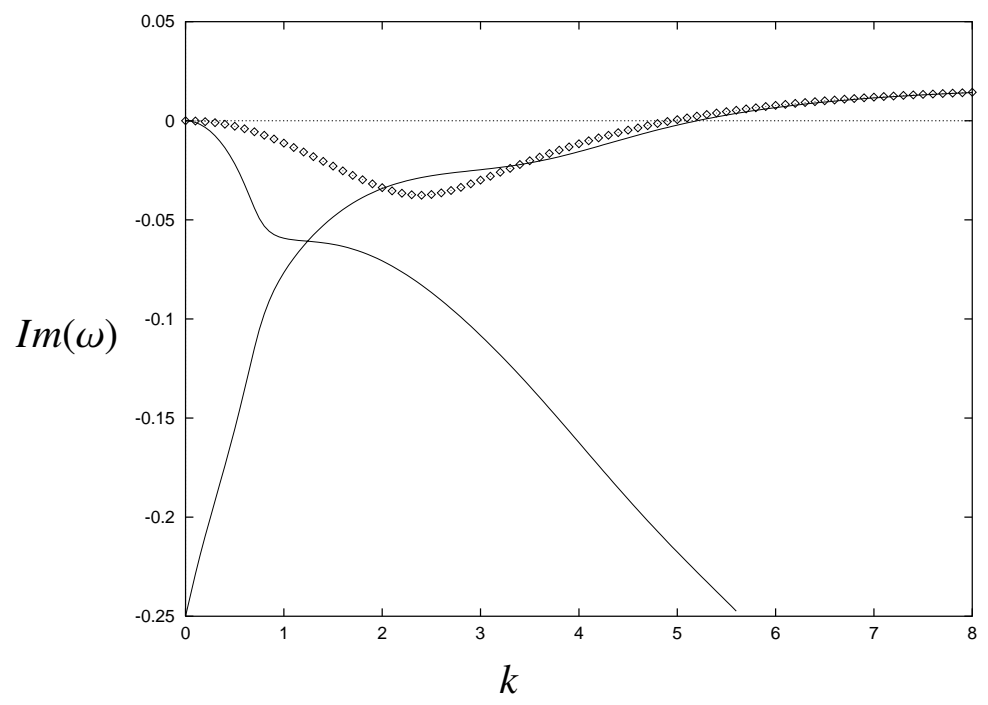

Figure 8: Growth rates plotted against $k$ for a case where long waves are stable and short unstable, $c=0.2, \kappa=0.5$, $W_{1}=0, W_{2}=4$. Inner fluid more elastic, fastest growing mode has $k=\infty$. The varicose mode (points) almost always has the higher growth rate; two different sinuous modes are shown (solid lines).

[2] K.P. Chen, Interfacial instability due to elastic stratification in concentric coextrusion of two viscoelastic fluids, J. Non-Newtonian Fluid Mech. 40 (2) (1991) 155-176.

[3] E.J. Hinch, O.J. Harris, J.M. Rallison, The instability mechanism for two elastic liquids being coextruded, J. Non-Newtonian Fluid Mech. 43 (2-3) (1992) 311-324.

[4] T.C. Ho, M.M. Denn, Stability of plane Poiseuille flow of a highly elastic fluid, J. Non-Newtonian Fluid Mech. 3 (1977) 179.

[5] P. Laure, H. Le Meur, Y. Demay, J.C. Saut, S. Scotto, Linear stability of multilayer plane Poiseuille flows of Oldroyd B fluids, J. Non-Newtonian Fluid Mech., (1996) submitted..

[6] J.M. Piau, N. El Kissi, F. Toussaint, A. Mezghani, Distortions of polymer melt extrudates and their elimination using slippery surfaces, Rheol. Acta 34 (1) (1995) 40-57.

[7] Y.Y. Renardy, Stability of the interface in two-layer Couette flow of Upper-Convected Maxwell liquids, J. NonNewtonian Fluid Mech. 28 (1988) 99-115.

[8] Y-Y Su, B. Khomami, Interfacial stability of multilayer viscoelastic fluids in slit and converging channel die geometries, J. Rheol. 36 (2) (1992) 357-387.

[9] Y-Y Su, B. Khomami, Purely elastic interfacial instabilities in superposed flow of polymeric fluids, Rheol. Acta 31 (1992) 413-420.

[10] C-S Yih, Instability due to viscosity stratification, J. Fluid Mech. 27 (1967) 337-352. 


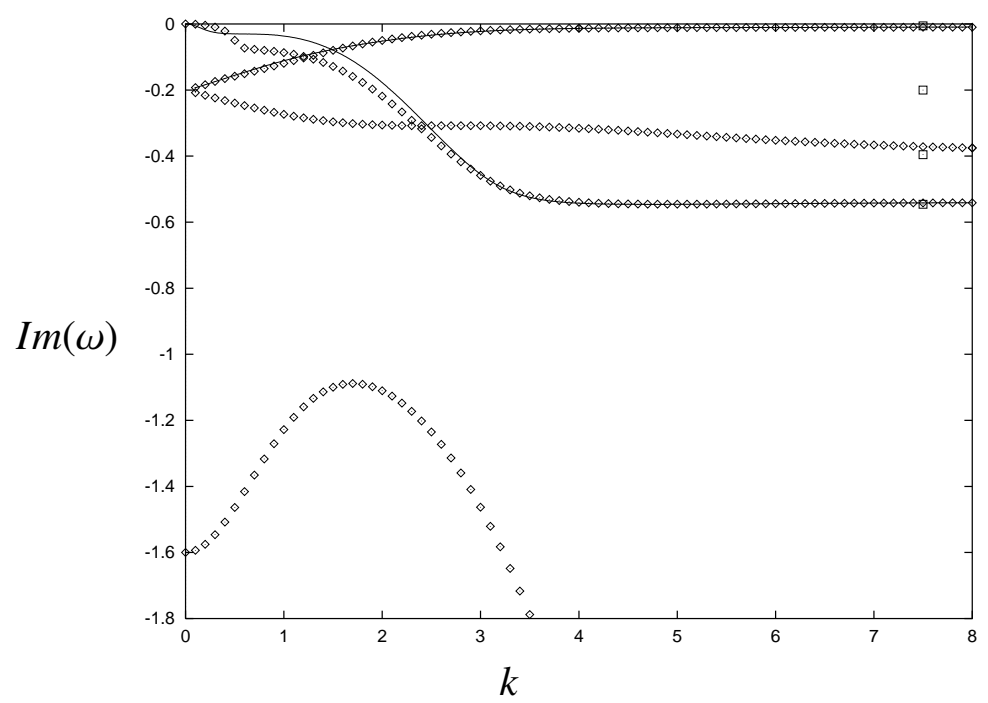

Figure 9: Growth rates plotted against $k$ for a case where both long and short waves are stable, $c=\infty$ (UCM), $\kappa=0.5$, $W_{1}=0, W_{2}=5$. Inner fluid more elastic; all modes decay. The sinuous modes are solid lines, the points are the varicose modes. In the short-wave limit, the dispersion relation is a polynomial which has five roots [7], four of which are marked here with small squares. The fifth has a decay rate of 5.8, and is not visible on the scale of this graph. For varicose modes we were unable to determine solutions along four of the known solution branches beyond $k_{c} \sim 3.5$. 